Voix et Images

volxetimages

\title{
Tel Quel ou les conditions d'émergence des Herbes rouges
}

\section{Pierre Milot}

Volume 13, numéro 2 (38), hiver 1988

Le propre du corps Roger Des Roches

URI : https://id.erudit.org/iderudit/200713ar

DOI : https://doi.org/10.7202/200713ar

Aller au sommaire du numéro

Éditeur(s)

Université du Québec à Montréal

ISSN

0318-9201 (imprimé)

1705-933X (numérique)

Découvrir la revue

Citer cet article

Milot, P. (1988). Tel Quel ou les conditions d'émergence des Herbes rouges. Voix et Images, 13(2), 317-323. https://doi.org/10.7202/200713ar d'utilisation que vous pouvez consulter en ligne.

https://apropos.erudit.org/fr/usagers/politique-dutilisation/ 


\section{Tel Quel ou les conditions d'émergence des Herbes rouges 1}

\section{par Pierre Milot, Université du Québec à Montréal}

La question de l'«influence» des modes et des revues parisiennes sur le champ littéraire québécois n'est pas nouvelle: du Manifeste du surréalisme au Refus global, des Temps Modernes à Parti pris, et de Tel Quel aux Herbes rouges, se profile une généalogie qui concerne directement les rapports de dépendance entre le champ littéraire français et le champ littéraire québécois.

Le problème avec cette notion d' «influence», c'est qu'elle manque de délimitation conceptuelle et qu'elle n'induit qu'une causalité discursive, alors que c'est l'ensemble des conditions sociales de production de ces modes et de ces revues qui sont au fondement des relations entre les deux champs littéraires. Lorsqu'on examine la façon dont cette problématique a été traitée jusqu'à ce jour, je pense qu'on peut isoler deux paradigmes: le paradigme du «mimétisme» et le paradigme de l'«intertextualité». Je me propose donc ici d'examiner brièvement les conséquences de l'usage argumentatif de l'un et l'autre de ces paradigmes, puis à mon tour je poserai l'usage d'un concept qui me paraît plus opératoire pour analyser le mode de réception de Tel Quel au Québec dans les années 1970.

Le paradigme du «mimétisme» consiste à accuser les écrivains de l'avantgarde littéraire regroupés aux Herbes rouges et à la Barre (Nouvelle Barre) du jour d'avoir imité (sinon plagié) les thèmes et les techniques des écrivains et des théoriciens réunis autour de la revue Tel Quel. L'exemple le plus récent d'assignation au procès du mimétisme provient d'un texte de Jean Larose publié dans Liberté et dont l'objectif était de disqualifier une certaine pratique d'écriture liée à l'imitation du modèle parisien 2 . Larose croit déceler une perte de sens et une dissimulation de la rupture moderne dans le mode d'importation des théories parisiennes en direction du champ littéraire québécois. L'accumulation des indices incriminants évoqués par Larose dans le transfert des leçons de maître Derrida aux désarrois de l'élève Brossard produit une inférence pour le moins discutable: jamais les contradictions ou les inconséquences du modèle ne sont relevées, seules les erreurs et les errances de l'épigone sont notées. Cette procédure de distinction a pour conséquence d'invalider l'entreprise de l'avant-garde

1 Communication présentée au Colloque sur les Herbes rouges, à l'UQAM, le 6 mars 1987.

2 Pour une lecture critique plus élaborée de ce texte, voir mon essai «la Légitimité offensée de l'avant-garde littéraire des années 1970», Voix \& Images, $\mathrm{n}^{\circ} 33$, printemps 1986 , p. 521-527. 
littéraire québécoise tout en laissant intactes les pratiques polymorphes de Tel Quel. Qu'en l'espace d'une décennie, Tel Quel soit passée des formalistes russes aux textes bibliques, du structuralisme au post-structuralisme, de la psychanalyse lacanienne à l'anti-psychanalyse des «machines désirantes» deleuziennes, de Mao Tsé-Toung à Jean-Paul II, du marxisme althussérien à la «nouvelle philosophie», du «refus du sujet» au discours amoureux, tout cela reste intouché par le paradigme du mimétisme: comme s'il n'y avait pas là les conditions de possibilité de l'indigence théorique des indigènes périphériques face aux maîtres penseurs de la métropole. De plus, le paradigme du mimétisme réduit la conduite des agents sociaux au climat ambiant de la contre-culture des années 1970 sans tenir compte des conditions institutionnelles de production, dont les changements opérés au sein du champ universitaire par les transformations des programmes d'études littéraires, comme cela s'est passé à l'Université du Québec à Montréal, au début des années 1970.

Quant au paradigme de l'intertextualité, il a été utilisé par l'avant-garde pour légitimer ses multiples sources d'influence et l'absorption des divers courants théoriques qui lui ont permis de se construire un agrégat discursif dont les postulats sont demeurés indéterminés. Ce paradigme a également autorisé le transfert de certains concepts de la «déconstruction» derridienne à la pratique textuelle: la «disparition» de la notion d'auteur et de celle d'œuvre était invoquée pour justifier les emprunts innommables et innombrables et pouvait servir de caution contre les accusations de mimétisme et de plagiat intentées par la critique littéraire du début des années 1970. Malheureusement pour l'avant-garde, ce paradigme s'est retourné contre elle: avec le «retour du sujet», l'auteur a repris ses droits et les œuvres ont réintégré les anthologies. De plus, le paradigme de l'intertextualité est d'un usage litigieux: lors de la polémique qui a opposé les Charron et Beaudet aux de Bellefeuille et Corriveau, à propos de la modernité et du sacré, les deux clans se sont mis à rationaliser les influences thématiques et techniques des uns et des autres en s'accusant mutuellement d'être des écrivains sous influence.

Les paradigmes du mimétisme et de lintertextualité ne nous permettent donc pas de saisir les conditions d'émergence et les stratégies discursives de l'avantgarde, pas plus qu'ils ne légitiment la représentation que les écrivains se font de leurs propres pratiques d'écriture. Pour comprendre l'avènement, le développement institutionnel et la consécration dont une revue comme les Herbes rouges fait l'objet, je pense qu'il faut construire l'économie des échanges littéraires en analysant les pratiques de l'avant-garde parisienne et les pratiques de l'avant-garde québécoise à travers le paradigme de l'homologie récurrente avec le parisianisme.

\section{Tel Quel: sous les pavés, la page}

Au milieu des années 1960 en France, paraissent deux ouvrages dont les auteurs allaient devenir les maîtres penseurs de toute une génération de professeurs et d'étudiants du système d'enseignement français: Pour Marx de Louis 
Althusser (1965) et Critique et vérité de Roland Barthes (1966) ${ }^{3}$. Ces deux livres braquent leurs armes discursives sur une cible commune: la conception universitaire courante (Althusser) et les interdits de la critique classique (Barthes). L'un et l'autre posent les énoncés d'une «nouvelle critique» axée sur la lecture scientifique des textes, une science de l'histoire qui permet de débusquer la problématique «enfouie» de toute pensée, afin de l'arracher des profondeurs de l'idéologie (Althusser), une science de la littérature donnant aux flottements du sens un statut scientifique... contre la cohérence psychologique de l'ancienne critique (Barthes). Somme toute, une approche structurale faisant apparaître l'ordre sous le désordre et révélant ce que la raison peut contenir de déraison. La science contre l'idéologie. Les sciences sociales (marxisme, structuralisme, psychanalyse, sémiologie) contre les humanités classiques de la Sorbonne. Une étude «Sur le jeune Marx» imposant d'autorité une coupure épistémologique dans l'approche scientifique des textes de Marx, une étude «Sur Racine» imposant d'autorité une rupture psychanalytique avec tout ce qu'on a appris à l'école sur Racine.

Fondée au début des années 1960 autour de Philippe Sollers et Jean-Pierre Faye (qui, suite à une rupture avec Sollers, lancera Change, une revue concurrente), Tel Quel est issue de la mouvance du «nouveau roman» dont elle se démarquera progressivement pour se rapprocher de la Nouvelle Critique (revue culturelle du Parti communiste français), tout en maintenant le cap sur un formalisme littéraire ajusté aux travaux des sciences du langage: sémiologie (Kristeva), redécouverte des formalistes russes (Todorov), grammatologie (Derrida), avec à l'arrière-plan le travail opéré par Lévi-Strauss (Anthropologie structurale) sur le Cours de linguistique générale de Ferdinand de Saussure appliqué à la recherche anthropologique (les «sociétés primitives» structurées comme un langage). Lacan en fera un usage discursif dont le profit symbolique restera longtemps à la hausse: l'inconscient est structuré comme un langage.

Autre indicateur de la percée du structuralisme et du déclin de la phénoménologie et du marxisme sartrien, la parution en 1966 de l'ouvrage de Michel Foucault, les Mots et les choses, dont les fondements épistémologiques et le style d'écriture ne sont pas sans rappeler la «coupure épistémologique» d'Althusser (concept emprunté à Gaston Bachelard, figure de proue de l'épistémologie française): la mort du sujet (Foucault) et le procès sans sujet (Althusser) seront en tout cas amalgamés par les rédacteurs de la revue Tel Quel qui feront d'Althusser et de Foucault (avec Barthes, Lacan et Derrida) les maîtres penseurs de leur Théorie d'ensemble.

Alors qu'autour des années 1965-1966, Althusser et Barthes avaient voulu imposer une «nouvelle critique» dans le champ des sciences sociales et dans le champ des études littéraires, à travers la montée du structuralisme, de la psychanalyse et de la linguistique (Lévi-Strauss, Lacan, Saussure), en Mai 68, ils se

3 Publiés respectivement aux éditions Maspéro et au Seuil. 
retrouvent mêlés d'un côté à toutes les tendances de l'extrême gauche politique (les groupes maoïstes et leur presse) et de l'autre au formalisme littéraire de la revue Tel Quel qui, tout en se réclamant des sciences du langage, prend position pour l'écriture et la révolution:

Par rapport à la littérature, ce que nous proposons veut être aussi subversif que la critique faite par Marx de l'économie classique... Toute écriture, qu'elle le veuille ou non, est politique. L'écriture est la continuation de la politique par d'autres moyens... L'écriture et la révolution font cause commune l'une donnant à l'autre sa recharge signifiante 4 .

Le marxisme althussérien opérant à partir de la double matrice de la lutte des classes (marxisme) dans la théorie (structuralisme), il autorisait les transferts d'énoncés politiques en énoncés littéraires et les déplacements de l'ordre du discours dans la pratique politique. Ce qui amènera Althusser à parler de «pratique théorique» et de «lutte des classes dans la théorie», légitimant par là l'enseignement et la recherche universitaires du moment que l'un et l'autre sont orientés par la «science marxiste» et se démarquent de «lidéologie bourgeoise».

Cette lutte pour l'autorité légitime de la science aura pour conséquence de subordonner les intérêts génériques du champ littéraire aux intérêts spécifiques du champ des sciences sociales. Conditions de production du formalisme telquelien, les sciences sociales en sont aussi les conditions de lisibilité. Ce formalisme dont on dira, lorsqu'il atterrira dans le champ littéraire québécois, qu'il est «illisible». Et c'est par ce biais de l'illisibilité que je voudrais maintenant aborder les conditions d'émergence des Herbes rouges à la fin des années 1960.

\section{Conditions de production et conditions de lisibilité}

Contrairement à la doxa véhiculée par une fraction de la critique littéraire (mais encouragée, il faut le dire, par certains écrivains formalistes eux-mêmes), les textes des Herbes rouges n'ont jamais été «illisibles», au sens qu'on a voulu donner à cette notion. Le débat sur la question de l'illisibilité est un cas de figure des rapports entretenus par un certain type de producteurs (l'avant-garde) et une certaine critique qui, soit ne partage pas les enjeux promus par ces producteurs et cherche à les disqualifier, soit n'est pas ajustée aux conditions de lisibilité que suppose l'habitus ${ }^{5}$ du lecteur formaliste. C'est un autre effet doxique de croire que les producteurs ont déposé dans les interstices de leur écriture des codes qui échapperaient à leurs conditions sociales de lecture. L'habitus du lecteur est la première condition de lisibilité du texte formaliste. Car décoder un texte formaliste, c'est construire une correspondance entre divers éléments lexicaux, syntaxiques et sémantiques surdéterminés par différents concepts importés des

4 Philippe Sollers, «Écriture et révolution», Théorie d'ensemble, Paris, Seuil, 1968 , p. $68,78-79$

5 Sur le concept d'habitus, voir Pierre Bourdieu, le Sens pratique, Minuit, 1980. 
sciences sociales des années 1960: du structuralisme au marxisme en passant par la sémiologie et la psychanalyse. C'est dans ce sens qu'on peut dire que les textes formalistes sont des textes écrits par des producteurs pour d'autres producteurs: c'est ce qui fait de l'avant-garde un champ de production restreinte. C'est aussi pour cela que les critiques littéraires qui sont en mesure de lire ces textes sont ceux qui disposent d'un habitus ajusté aux mêmes stratégies institutionnelles et esthétiques que les producteurs.

Ce qui m'amène à énoncer le paradoxe suivant: le texte formaliste tel que pratiqué par certains écrivains des Herbes rouges dans les années 1970 était tout aussi instituant que les poèmes de la génération de Parti pris. Pour ne prendre qu'un exemple parmi d'autres: Au «sujet» de la poésie de Charron relevait tout autant du structuro-marxisme que l'Afficheur hurle de Chamberland avait pu relever du socialisme décolonisateur.

Et ici, je précise que définir les conditions de production et de lisibilité d'un texte formaliste à travers le structuro-marxisme n'entraîne pas une réduction du texte à sa seule dimension institutionnelle: les conditions de lisibilité d'un texte ne s'épuisent pas dans son processus institutionnel, mais ce processus n'en demeure pas moins la condition de possibilité du texte formaliste. Sa condition de possibilité, non sa forclusion.

\section{Économie poétique des Herbes rouges}

Quand on examine le contenu des premiers numéros des Herbes rouges (de même que celui de la Barre du jour), on se rend compte que l'effet de mode Tel Quel n'a pas encore atteint le champ littéraire québécois. Aux Herbes rouges, tout comme à la Barre du jour, on fait cohabiter les poètes de la génération de l'Hexagone (Miron) et de Parti pris (Chamberland) avec de nouveaux noms (Roger Des Roches, Louis-Philippe Hébert): rien dans tout cela qui ne laisse anticiper une quelconque «révolution du langage poétique». Plutôt une sorte de consensus tranquille entre les poètes institués et des nouveaux entrants dont les poèmes n'ont rien des pavés lancés contre la vitrine de l'institution littéraire par les écrivains et les théoriciens de Tel Quel en France.

Les Herbes rouges et la Barre du jour échangent leurs auteurs dans une complicité dont l'enjeu est leur stratégie d'émergence: revues faisant la promotion d'une «nouvelle écriture» sans pour autant rompre violemment avec la génération précédente. La Barre du jour consacre un numéro à Roland Giguère et les Herbes rouges publient côte à côte Jacques Brault, Paul-Marie Lapointe, François Charron et Nicole Brossard.

François Charron et Roger Des Roches ont joué un rôle stratégique dans le mode de réception et d'appropriation des thèmes et des techniques de Tel Quel: leur texte «Notes sur une pratique» paru dans le numéro 29 (été 1971) de la Barre du jour constitue la première véritable retraduction du telquelisme dans le champ littéraire québécois. Car même si Nicole Brossard avait introduit une 
pratique formaliste d'écriture, elle était toujours demeurée rétive à fournir les postulats théoriques de son entreprise. La préface écrite par Charron pour le deuxième livre de Roger Des Roches, l'Enfance d'yeux (1972), constituait un véritable mode d'emploi de la «pratique textuelle» qui sera perpétuée par d'autres écrivains des Herbes rouges dont André Roy et Normand de Bellefeuille6. Charron y proclamait que

l'écriture ici inscrite [...] montre ce que toute forme poétique a d'inacceptable tant qu'elle ne dénonce pas sans cesse son processus de production dans le code linguistique.

Charron y précisait qu'il fallait démasquer la poésie en tant que discours idéologique et que cette attaque était perpétrée contre tout un édifice littéraire bien rodé. Et pourtant, cet ouvrage de Roger Des Roches peut être considéré comme une des performances formalistes les plus caractéristiques de la pratique textuelle de l'époque.

Avec la création de Stratégie à l'hiver 1972 et celle de Hobo-Québec en décembre de la même année, la situation des revues d'avant-garde va changer: une nouvelle concurrence va s'installer, qui n'est pas immédiatement repérable, mais qui aura des répercussions à moyen terme. D'une part, Stratégie et HoboQuébec tiennent un discours beaucoup plus «agressif» en ce qui concerne la littérature, d'autre part, l'une et l'autre de ces nouvelles revues cherchent à se distinguer des revues déjà existantes par le biais de procédés discursifs marqués: les pratiques «signifiantes» pour Stratégie, les pratiques «contre-culturelles» pour Hobo-Québec.

Puis ce sera l'arrivée de Brèches (1973), Champs d'application (1974) et Chroniques (1975). Les Herbes rouges ont pu s'adapter à cette concurrence par un nouveau dispositif: le numéro d'auteur se substituant au numéro collectif. C'est le numéro d'auteur qui permettra aux Herbes rouges de se situer au croisement des diverses revues et des différents écrivains que ces dernières propulsaient dans le champ littéraire. Ainsi, entre les Problèmes du cinématographe de Des Roches et N'importe qu'elle page d'André Roy, la revue a publié Snack-bar de Lucien Francœur, puis suite au Space-Opera de Des Roches, le $\mathbf{4 X 4}$ de Patrick Straram suivi du Clitoris de la fée des étoiles de Denis Vanier, sans compter le premier recueil de Philippe Haeck, Nattes et Machine-t-elle de Yolande Villemaire. Tous ces auteurs étaient membres des comités de rédaction (ou collaborateurs réguliers) de la Barre du jour, Stratégie, Hobo-Québec ou Chroniques et représentaient des tendances concurrentes et conflictuelles qui ont généré de nombreuses polémiques. De plus, les frères Hébert (directeurs des Herbes rouges), en devenant directeurs d'une collection de poésie («Lecture en vélocipède») aux éditions L'Aurore que venait de fonder Victor-Lévy Beaulieu en 1974, pourront continuer la promotion de leurs auteurs maisons (les Charron, Des Roches, Roy et Haeck).

6 «La Matière du livre», préface de François Charron à l'Enfance d'yeux, de Roger Des Roches, Éditions du Jour, Montréal, 1972, p. 7-11. 
C'est dans la seconde moitié des années 1970 qu'émergera l'écriture féministe dont la Barre du jour deviendra la principale génératrice discursive à partir du numéro Femmes et langage en 1975. Axée sur les modalités énonciatives des textes publiếs par les Éditions des Femmes (dont ceux d'Hélène Cixous) et les thèses de la philosophie post-structuraliste (Derrida, Deleuze, Lyotard), cette écriture «de femmes» a contribué à restructurer les enjeux institutionnels et esthétiques de l'avant-garde littéraire. Aux Herbes rouges, ce sont surtout les Corriveau et les de Bellefeuille qui semblent être devenus les compléments directs des écritures féministes de la BJ (l'un et l'autre deviendront d'ailleurs respectivement directeur et secrétaire de rédaction de la Nouvelle Barre du jour dans les années 1980). Bien que les Herbes rouges aient publié quelques femmes (Madeleine Gagnon, France Théoret) pendant cette montée du féminisme littéraire, ce sont surtout les écrivains (au masculin pluriel) qui vont chercher à renouveler les thèmes et les techniques de la maison. C'est l'époque du Roland Barthes par Roland Barthes et des Fragments d'un discours amoureux à laquelle on donnera ici le nom de «retour du lyrisme».

Quant aux années 1980, elles sont nettement dominées par les activités incessantes de la Nouvelle Barre du jour dont les stratégies de promotion se traduisent par l'organisation de multiples colloques publicisés par le Devoir et qui donneront lieu à une pléthore de polémiques entre la NBJ et Liberté (dont le texte de Larose n'est qu'une suite logique) d'une part, et entre divers écrivains liés à la fois à la NBJ et aux Herbes rouges (si l'on excepte Charron et Beaudet dont les noms sont principalement associés aux Herbes rouges) d'autre part. C'est l'époque où Bernard-Henri Lévy médiatise le Testament de Dieu, où Tel Quel devient l'Infini et à laquelle on donnera ici le nom de «retour du sacré».

Il faut saisir le débat entre la modernité et le sacré comme un état de la concurrence entre les Herbes rouges et la Nouvelle Barre du jour: mais ce qu'il faut comprendre, c'est qu'il s'agit d'une saine concurrence entre deux revues d'avant-garde qui sont devenues de véritables instances de légitimation de l'institution littéraire québécoise, et dont les auteurs sont des entrepreneurs autonomes sur le marché littéraire des années 19807. Mais peut-on parler pour autant d'autonomie littéraire entre le marché parisien et le marché québécois? Pour le savoir, il faudra analyser les effets institutionnels et esthétiques des prochaines encycliques post-modernes de l'Infini sur les «pratiques modernes» des... Herbes rouges.

7 Sur cet aspect du conflit modemité/sacré, voir mon texte «la Camera obscura du post-modernisme», Volx \& images, $n^{\circ} 34$, automne 1986, dans lequel je traite plus particulièrement du rôle stratégique joué par André Beaudet dans la promotion de ce conflit symbolique, et «Qui a peur de l'intellectuel en 1987?», Volx \& images, $n^{\circ} 36$, printemps 1987, où j'analyse la dénégation de ce conflit dans le livre de Hugues Corriveau et de Normand de Bellefeuille, ג̇ double sens, publié aux Herbes rouges. Ces textes, ainsi que celui sur Jean Larose, ont été repris dans un ouvrage à paraître à l'Hexagone: la Camera obscura du postmodernisme (printemps 1988). 